\title{
Behindertenrecht und Behindertenpolitik in der Kommune - ein Blick in die Rechtsprechung
}

\author{
Stephan Rittweger
}

I. Einleitung

II. Entwicklungen in der neueren Rechtsprechung

1. Kein Auto zulasten der Sozialhilfe

2. § 71 SGB XII: Kulturelle Teilhabe, kein Anspruch auf Theaterbesuch

3. Kein Sport-Rollstuhl - Krankenversicherung

4. Inklusionsziel Bildung

5. Kein Cialis zulasten der Gesetzlichen Krankenversicherung

III. Zusammenfassung und Ausblick

\section{Einleitung}

Die Gerichte haben als Dritte Gewalt im Staate die klassische Aufgabe, die anderen Staatsgewalten zu kontrollieren. Die Sozialgerichte haben den speziellen Auftrag, die Sozialleistungsträger zu überprüfen und sie immer wieder anzuhalten, dass jedem Berechtigten die ihm zustehenden Sozialleistungen in zeitgemäßer Weise, umfassend und zügig zukommen vgl. § 17 SGB I. Dabei hat das Bundesverfassungsgericht die Sozialgerichte schon manches Mal daran erinnert, dass sie sich schützend und fördernd vor die Rechtsgüter der Betroffenen stellen sollen. Diese Aufgabenstellung erfordert, Gerichtsverfahren einem zeitnahen Ende durch gütliche Einigung zuzuführen. Klagen über mehrere Instanzen und Jahre hinweg schaden oft mehr als sie den Betroffenen nutzen. Die weit überwiegende Zahl der Verfahren vor den Sozialgerichten endet deshalb unstreitig, Anerkenntnisse und Vergleiche beenden weitaus mehr Verfahren als Urteile und Beschlüsse. Das ist der Grund, warum ein Überblick über die sozialgerichtliche Rechtsprechung keineswegs vollständig abbildet, wie Sozialbehörden und Sozialgerichte zur Inklusion stehen.

Die Aufgaben der sozialgerichtlichen Rechtsprechung erschöpfen sich aber nicht in der Schaffung von Rechtsfrieden im Einzelfall. An vielen Stellen des Sozialgesetzbuches hat der Gesetzgeber unbestimmte Rechtsbegriffe verwendet, ja verwenden müssen. Deren Inhalte zu bestimmen ist eine weitere bedeutsame Aufgabe der Gerichte. Ihre 
Entscheidungen konkretisieren die Regelungen der Gesetze; auch bilden sie das Recht fort. Hier haben die Sozialgerichte einen Definitionsauftrag und eine Definitionshoheit.

Dies vorausgeschickt ist festzustellen, dass die Rechtsprechung bereit ist, sich auf die UN-Behindertenrechtskonvention als normative Handlungsdirektive einzustellen. Die Sozialgerichte lassen nach und nach erkennen, welche konkreten Rechtsansprüche aus dem abstrakten Ziel der Inklusion folgen - und wie die Balance zwischen Inklusion und Finanzlast zu finden ist.

\section{Entwicklungen in der neueren Rechtsprechung}

\section{Kein Auto zulasten der Sozialhilfe}

In einem Fall, über den das Bayerische LSG zu entscheiden hatte, machte ein 53 Jahre alter Kläger (GdB 100, Merkzeichen G, B, RF und aG) beim Träger der Sozialhilfe geltend, er benötige besondere Hilfen, um kulturelle Veranstaltungen besuchen zu können. Dazu sei er wegen seiner Wohnlage und seiner Behinderung auf einen PKW angewiesen. Er verlangte daher Kostenerstattung für Reparatur, TÜV, Versicherung und Benzin nach $\S \S 53,54$ SGB XI iVm der Eingliederungshilfe-VO.

Mit Urteil vom 26.02.2010 ${ }^{1}$ erkannte das Gericht einen Anspruch des Klägers auf Teilhabe an kulturellen Veranstaltungen dem Grunde nach an. Die KFZ-Kosten seien aber nicht zu übernehmen, denn der Behindertenfahrdienst des Bezirks ermögliche es dem Kläger in noch ausreichendem Umfang, am Leben in der Gemeinschaft teilzunehmen. Der Kläger könne auf diesem Wege einmal monatlich kulturelle Veranstaltungen besuchen und soziale Kontakte pflegen. Auch sei die Inanspruchnahme von öffentlichen Verkehrsmitteln mithilfe der Ehefrau zumutbar (§ 2 Abs. 1 SGB XII).

Die UN-Behindertenrechtskonvention wird in dieser Entscheidung nicht benannt.

\section{2. §71 SGB XII: Kulturelle Teilhabe, kein Anspruch auf Theaterbesuch}

In einem weiteren Fall ging es um einen 1938 geborenen Kläger, der Grundsicherung im Alter bezog. Er bat um Auskunft, welche Möglichkeiten die Altenhilfe nach dem SGB XII eröffne, ihn zu unterstützen. Der Gesetzgeber habe bei § 71 SGB XII an eine finanzielle monatliche Unterstützung zum Besuch von Veranstaltungen oder Einrichtungen gedacht. Auch die Gewährung von Reisebeihilfen bis hin zu einem Erholungsurlaub sei in $\S 71$ SGB XII enthalten.

1 Az. L 8 SO 55/09. 
Der achte Senat des Bayerischen LSG hat mit Urteil vom 26.02.2010² einen Anspruch auf Teilhabe am kulturellen Leben dem Grunde nach anerkannt, im konkreten Fall aber für den Kläger verneint. Zu den persönlichen Bedürfnissen des täglichen Lebens gehörten im Rahmen der Altenhilfe gem. § 71 SGB XII in vertretbarem Umfang auch Beziehungen zur Umwelt und eine Teilnahme am kulturellen Leben ( $\$ 27$ Abs. 1 S. 2 SGB XII). Die Leistungen der Altenhilfe seien in einem Katalog nach $\S 71$ Abs. 2 Nr. 5 SGB XII konkretisierend derart beschrieben, dass insbesondere Leistungen zum Besuch von Veranstaltungen oder Einrichtungen, die der Geselligkeit, der Unterhaltung, der Bildung oder den kulturellen Bedürfnissen alter Menschen dienen, in Betracht kämen. Damit komme durchaus ein individueller Einzelanspruch in Betracht. Voraussetzung für derartige Ansprüche sei aber das konkrete Vorliegen einer entsprechenden Infrastruktur und eines entsprechenden Angebots sowie deren Geltendmachung. Insoweit fehle es dem Kläger aber zur unmittelbaren Geltendmachung von Ansprüchen an einem Rechtsschutzbedürfnis, weil ihm keine konkreten Vergünstigungen versagt worden seien.

Zwar seien die Bedarfe abweichend von $\S 28$ Abs. 1 S. 2 SGB XII festzulegen, wenn im Einzelfall ein Bedarf ganz oder teilweise anderweitig gedeckt sei oder unabweisbar sei und der Höhe nach erheblich von einem durchschnittlichen Bedarf abweiche. Es sei aber nicht ersichtlich, wie hier der Bedarf des Klägers unabweisbar seiner Höhe nach erheblich vom üblichen Bedarf abweiche. Dieser könne jedenfalls nicht dadurch begründet werden, dass bestimmte kulturelle Bedürfnisse des Klägers aufgrund seiner bisherigen Lebensführung besonders ausgeprägt vorhanden seien.

Die UN-Behindertenrechtskonvention wird auch in dieser Entscheidung nicht benannt.

\section{Kein Sport-Rollstuhl-Krankenversicherung}

In einem vom BSG mit Urteil vom 18.05.2011³ entschiedenen Fall begehrte der Kläger einen Sportrollstuhl, um am Behinderten-Basketball im Verein teilnehmen zu können. Der Rollstuhl, mit dem ihn die Krankenkasse versorgt hatte, war zwar für den Schulsport ausreichend, nicht aber für das Basketballspiel im Verein.

Das BSG hat einen Anspruch auf den Rollstuhl für den Vereinsbasketball nach § 33 Abs. 1 Satz 1 SGB V verneint. Beim mittelbaren Behinderungsausgleich schulde die Kasse nur einen Basisausgleich, nicht aber einen Ausgleich im Sinne des vollständigen Gleichziehens mit den letztlich unbegrenzten Möglichkeiten eines gesunden Menschen (Bestätigung der bisherigen Rechtsprechung zum unmittelbaren und mittelbaren Behinderungsausgleich ${ }^{4}$ ).

2 Az. L 8 SO 129/09.

3 Az. B 3 KR 10/10 R.

4 Vgl. BSG Urteil vom 16.9.2004 - B 3 KR 20/04 R - C-Leg II. 
Durch das Übereinkommen der Vereinten Nationen über die Rechte von Menschen mit Behinderungen ergebe sich keine weitere Leistungsverpflichtung der Krankenkassen. Aus der UN-Konvention resultiere kein subjektiv-öffentliches Recht, ein konkretes und der persönlichen Mobilität dienendes Hilfsmittel von einem bestimmten Leistungsträger verlangen zu können. Die Bundesrepublik trage dem Zweck der UN-Konvention, den vollen und gleichberechtigten Genuss aller Menschenrechte und Grundfreiheiten durch alle Menschen mit Behinderungen zu fördern, zu schützen und zu gewährleisten sowie die Achtung der ihnen innewohnenden Würde $\mathrm{zu}$ fördern (Art. $1 \mathrm{UN}$ Konvention) ausreichend durch das gegliederte Leistungssystem des SGB und insbesondere durch dessen Neuntes Buch (Rehabilitation und Teilhabe behinderter Menschen - SGB IX) Rechnung. Weitergehende Einzelansprüche würden - zumindest im SGB V - durch die UN-Konvention nicht begründet.

Die UN-Behindertenkonvention wird in den kommenden Jahren zu einem Paradigmenwechsel weg von der Funktionsstörung hin zur Teilhabestörung führen. In diesem Rahmen wird es wohl dazu kommen, dass die von der Rechtsprechung entwickelte Unterscheidung von unmittelbarem und mittelbarem Behinderungsausgleich auf den Prüfstand gestellt wird.

\section{Inklusionsziel Bildung}

In einem anderen Fall, der dem Bayerischen LSG vorlag, ging es um zwei gehörlose sechsjährige Mädchen. Sie besuchten die Grundschule und nicht, wie vom Sozialhilfeträger gewollt, die Förderschule. Für den Inklusionsunterricht an der Regelschule benötigten sie die Hilfe von Gehörlosen-Sprachdolmetschern. Deren Kosten zu tragen, versagte der Sozialhilfeträger.

Mit seinen Beschlüssen vom 02.11.20115 konstatierte das LSG, Inklusion werde nicht vordergründig durch den Besuch der Regelschule hergestellt. Bildung als Ziel des Schulbesuchs sei mehr, als nur die funktionellen Fertigkeiten zu erwerben, wie Lesen, Rechnen und Schreiben. Das Ziel der integrativen Teilhabe an der Gemeinschaft als individuell entwickelte Persönlichkeit (Inklusion) lasse sich im konkreten Falle eher an der Förderschule erreichen.

Auch wenn der Senat entschieden hat, die UN-Behindertenrechtskonvention führe nicht zu einem unmittelbaren Rechtsanspruch auf Eingliederungshilfe, können beide Entscheidungen als Meilenstein gesehen werden. Das Gericht wendet nämlich die UNBehindertenrechtskonvention an. Und es stellt klar, dass sich ein angemessener Bedarf bei einer Eingliederungsmaßnahme nicht schon wegen Mehrkosten verneinen lässt. Ein Hauptsatz der Entscheidungen besagt nämlich, dass unter angemessener Schulbildung

5 Az. L 8 SO 165/11 B ER und L 8 SO 164/11 B ER. 
iSd $\S \S 53,54$ SGB XII alles zu verstehen ist, was der Erreichung des Ziels, der Integration in die Gesellschaft, dient.

Beide Entscheidungen waren im vorläufigen Rechtsschutz ergangen. Mittlerweile ist ein Hauptsacheverfahren durch gerichtlichen Vergleich beendet worden.

\section{Kein Cialis zulasten der Gesetzlichen Krankenversicherung}

In einem Urteil vom 6.3.20126 hatte das BSG über das Begehren eines Klägers auf Gewährung des von der Versorgung durch die gesetzliche Krankenversicherung ausgeschlossenen Arzneimittels „Cialis“ zu entscheiden. Der Kläger litt an einer erektilen Dysfunktion als Folge einer chronisch progredienten Multiplen Sklerose und sah in der Nichtgewährung des Arzneimittels unter anderem eine Verletzung des Art. 25 UN-BRK (Recht auf Gesundheit).

Im Ergebnis hat das BSG einen Anspruch auf besagtes Arzneimittel zwar verneint. Der gesetzliche Leistungsausschluss nach dem SGB V kollidiere weder mit Art. 25 UNBRK noch verstoße er gegen das Diskriminierungsverbot des Art. 5 Abs. 2 UN-BRK oder des Art. 3 Abs. 3 Satz 2 GG, weil er nicht an eine Behinderung im konventionsoder verfassungsrechtlichen Sinne anknüpfe, sondern alle Fälle der Erkrankung erfasse. Dessen unbeschadet hat sich das BSG aber hier sehr ausführlich Rang und Bedeutung der UN-BRK innerhalb der deutschen Rechtsordnung verankert.

\section{Zusammenfassung und Ausblick}

Der Blick auf diese fünf Entscheidungen zeigt verlässlich, dass die Sozialgerichte den Rechtsanspruch auf Inklusion nicht nur erkennen, sondern umsetzen und im Einzelnen konkretisieren. Ob Hilfen zur Teilhabe

- erforderlich,

- angemessen und

- notwendig sind,

wird künftig mehr und mehr nach den Grundsätzen des Rechtsbegriffes der Inklusion entschieden werden. Hier stellen sich die Sozialgerichte ihren Aufträgen zur Konkretisierung und zur Fortbildung des Rechts der Behinderten. Definitionsauftrag und Definitionshoheit bedeutet aber auch für die Sozialgerichte eine besondere Definitionsverantwortung. Der gilt es, gerecht zu werden.

6 Az. B 1 KR 10/11 R. 


\section{Behindertenrecht und Behindertenpolitik in der Kommune aus kommunaler Sicht}

\section{Helmut Kneppe}

$\begin{array}{ll}\text { I. Einleitung } & 141\end{array}$

1. Sozialraum 141

2. Personenzentrierung 142

3. Aufgabenverständnis der Kommunen 142

II. Kommentierung aus kommunaler Sicht 143

1. Ausgangspunkte der Ausführungen 143

2. Die Realisierung - aus dem Blickwinkel eines kommunalen Praktikers $\quad 144$

III. Fazit

\section{Einleitung}

Der Kommentar erfasst folgende Aspekte: Unter dem Eindruck der bisher vorgetragenen Tagungsbeiträge insgesamt werde ich in aller Kürze auf drei Stichworte eingehen. Diese Stichworte spreche ich aus meinem Blickwinkel ganz praktischer Planungsund Umsetzungsbelange im Sozialraum an. Hierbei bitte ich um ihr Verständnis für die eben wegen der Kürze an der Oberfläche gebliebene Argumentation:

\section{Sozialraum}

Aus meiner von praktischen Zusammenhängen geprägten Sicht ist der Sozialraum für den betroffenen Menschen (Adressaten einer sozialen Leistung) in der Regel keine universell und über bestimmte Koordinaten objektiv zu beschreibende absolute Fläche bzw. kein absoluter Raum. Trotzdem ist ein Sozialraum natürlich räumlich zu verstehen und hat insoweit auch die Aufgabe, räumliche Bezüge und Zusammenhänge herzustellen. Er ist in seiner konkreten Gestaltung immer abhängig von objektiven und subjektiven Rahmenbedingungen des einzelnen (behinderten) Menschen und seinem individuellen (Lebens-) Umfeld. 